\title{
Growth and Body Movement Analysis Using Image Processing in Early Stages of Chick Embryos Under Hypoxic Environment
}

\author{
Asahi Yokota ${ }^{\mathrm{a}}$, Koki Tenma ${ }^{\mathrm{a}}$, Yuya Chiba ${ }^{\mathrm{b}}$, Kenji Moriya $^{\mathrm{a}^{*}}$ \\ aDepartment of Production Systems Engineering, National Institute of Technology Hakodate College, \\ Tokura-cho 14-1, Hakodate City, Hokkaido, 042-8501, Japan \\ ${ }^{\mathrm{b} S}$ Support Center for Engineering Education, National Institute of Technology Hakodate College, \\ Tokura-cho 14-1, Hakodate City, Hokkaido, 042-8501, Japan \\ *Corresponding Author: moriya@ @akodate-ct.ac.jp
}

\begin{abstract}
Growth delay and unusual body movement was reported in hypoxia environment. We focused on those two phenomena and investigated. About growth delay, we measured size of chick embryo using image processing in $21 \%, 15 \%$ and $10 \%$ oxygen concentration environments. Chick embryos grew up that is same way on all environments until 110 hours. But growth level changed after 110 hours on each environment. On $21 \%$ oxygen concentration environment, chick embryos grew up rapidly. On $15 \%$, growth speed became slow. On $10 \%$, growth was stopped and this chick embryo died. This tendency is similar to change of amount of body movements in hypoxia environment. About body movement, we already have body movement analysis program. Thus, we attempted improvement of analysis program. Existing program is image processing program that analyze movement of chick embryos head. We developed new analysis program that analyze center of gravity of chick embryo for analyze whole body movement of chick embryo. As a result of analysis, center of gravity did not move. Therefor we think that center of gravity is axis of body movement. In future tasks, more experiments should be required to verify whether this tendency of embryonic growth is general or not. In addition, the center of gravity was not detected accurately because blood vessels of embryo were detected simultaneously when system detected the center of gravity, which means we have to extract only embryonic body by the image processing.
\end{abstract}

Keywords: chick embryos, embryonic growth, body movement, image processing.

\section{Introduction}

In the laboratory which we belong, body movement of chick embryo has been investigated instead of human fetus. Body movement is important parameter on growth. We were reported that image processing is valid for analysis of body movement ${ }^{(1,2)}$. In the previous research, body movement pattern in usual environment was elucidated to a certain extent. Also growth delay and unusual body movement was reported in hypoxia environment ${ }^{(3)}$. We focused on Growth delay and unusual body movement, and we tried that measurement of chick embryo size and improvement of body movement analysis program.

\section{Method of experiment}

\subsection{Incubation, oxygen environment and shoot}

We used broiler egg. Egg was incubated in environment that temperature was $38^{\circ} \mathrm{C}$, humidity was $60 \%$, usual oxygen concentration. After 73 hours, egg was taken a movie in usual oxygen concentration and hypoxia environment until 144 hours. About oxygen concentration, we experimented in environment that $21 \% \mathrm{O}_{2}, 15 \% \mathrm{O}_{2}$, $10 \% \mathrm{O}_{2}$. Taking a movie was done as follows. After incubated 72 hours, we drill $1.5 \mathrm{~cm}$ hole in egg. If egg is fertilized egg, set spacer and CCD camera, and start record. 
We stopped record at after incubated 144 hours. Movie recoded by $\mathrm{CCD}$ camera is saved to computer by capture board.

\subsection{Analysis of growth}

Fig.1 shows procedure of analysis of growth. Fist frame was picked every 1 hour. Embryo's contour was extracted by that frame. We fill white on inside contour. We calculated embryo size by counted white pixels. We analyzed movies of all oxygen concentration environments $(21 \%, 15 \%, 10 \%)$. When extract contour, we used Canny method. Canny method decide contour by threshold. Appropriate threshold was different each frame. So we adjust threshold manually. Fig. 2 show success example(a) and failure example(b) at extracted contour.

\subsection{Analysis of body movement}

We already have body movement analysis program ${ }^{(3)}$. That program analyzes chick embryos head movement by template matching because chick embryos head moves most violently. In this study, we made new body movement analysis program that analyze motion of center of gravity to embryo as a whole. Fig.3 show procedure of new program.1 frame was picked from movie. We searched chick embryo from that frame by template matching. Matching aria was converted grayscale. Only embryo was identified by binarization. We analyzed motion of center of gravity using binarization image. The above procedure was done once out of 10 frames. Template was updated every 5 minutes because chick embryo grows. About amount of

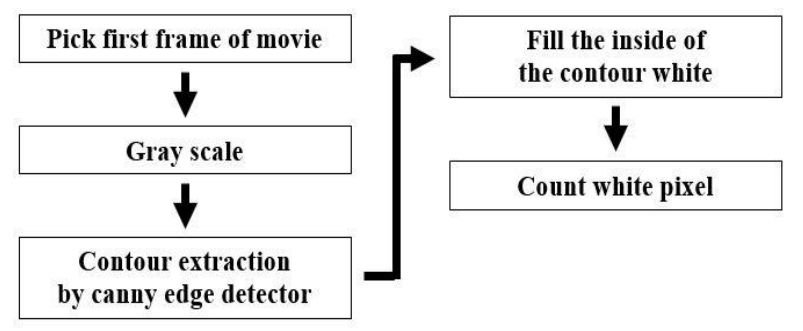

Fig.1. Procedure of growth delay

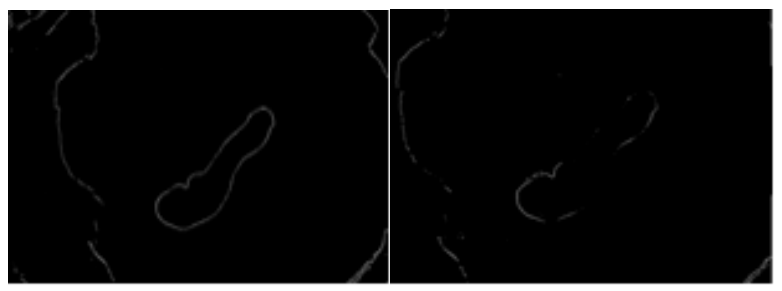

(a)Success example

(b)Failure example

Fig.2. Image of contour extraction

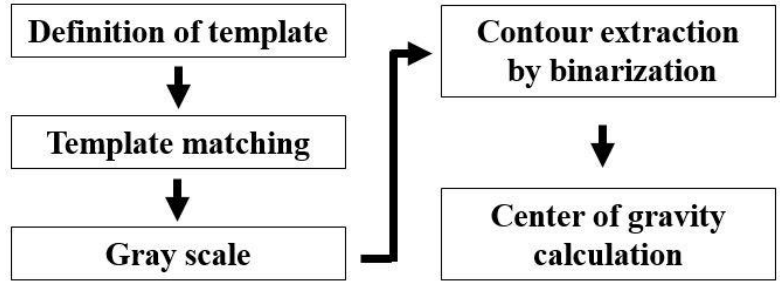

Fig.3. Calculation of chick position

body movement, we calculated amount of relative movement because amount of relative movement is more important than absolute position.

\section{Results and discussion}

\subsection{Analysis of growth}

We analyzed in 3 patterns of environment that oxygen concentrations are $21 \%, 15 \%$ and $10 \%$. Fig.4 shows result. Raised chick embryo in $10 \% \mathrm{O}_{2}$ environment was dead after 128 hours. We show result until 130 hours to compare. Until 110 hours, growth speed was same on each environment. On $21 \%$ oxygen concentration environment, chick embryo grew up rapidly after 110 hours. On $15 \%$ oxygen concentration environment, chick embryo grew up slowly than embryo that grew up in $21 \%$ oxygen concentration. On $10 \%$ oxygen concentration environment, chick embryo did not grow up and we confirmed death of embryo.

We understood that chick embryos growth in hypoxic environment is very slowly. We think that chick embryo could not breathe satisfactorily by decline oxygen concentration. Therefore, growth of embryo was affected.

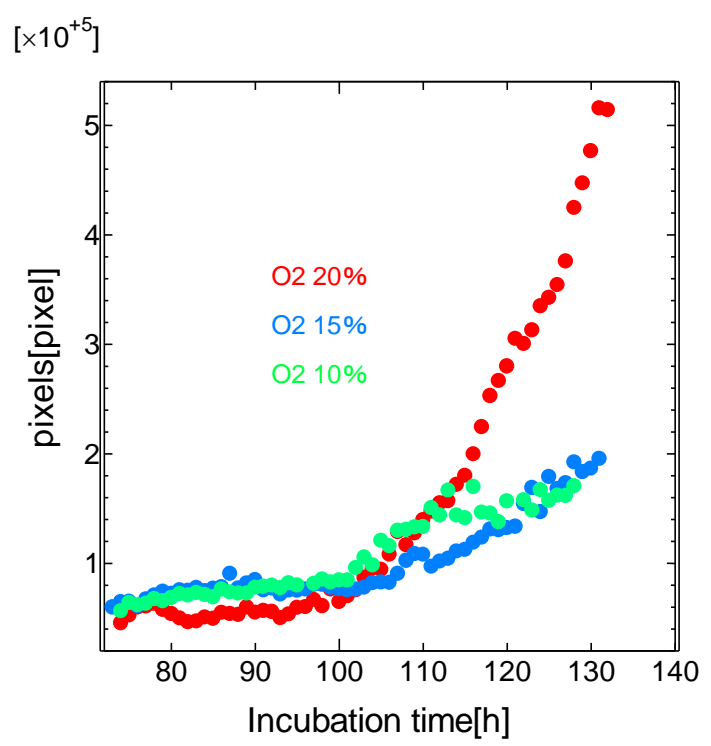

Fig.4. Growth comparison 


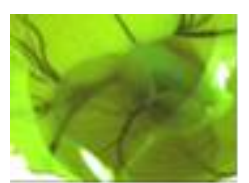

(a)usual

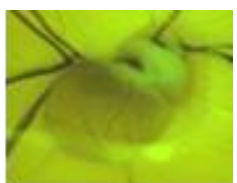

(b) $15 \% \mathrm{O}_{2}$

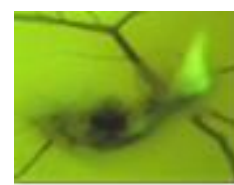

(c) $10 \% \mathrm{O}_{2}$
Fig.5. Embryos after 120 hours

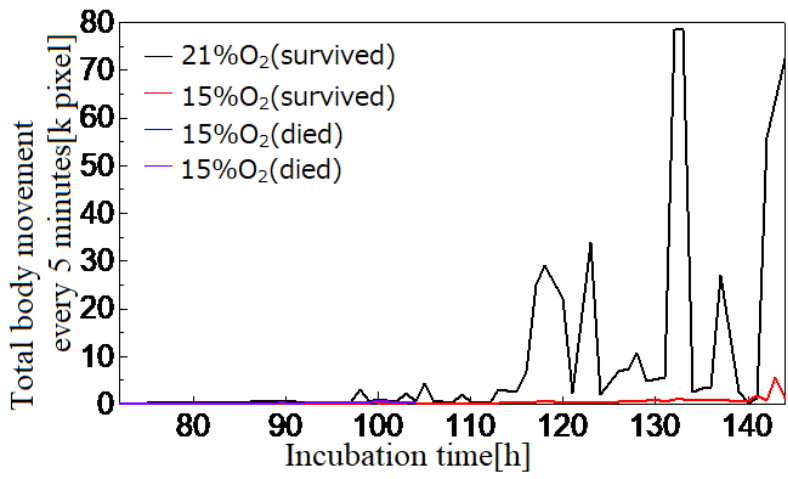

Fig.6. Total amount of body movement

(Unpublished data)

Fig.5. show embryo of each oxygen environment after 120 hours. We confirmed that growth of embryos amniotic membrane was delayed. Amniotic membrane covers the embryo to protect impact and to breathe easily. Because of these reasons, we think that embryo died by slight impact, or due to difficult of respiration.

In addition, in previous research, body movement of chick embryo in hypoxic environment is a few amount than usual environment. Fig.6 show total amount of body movement at every 5 minutes. Body movement was increase from after 110 hours. This timing resembles timing that chick embryo grew up rapidly. It is cause that when embryo's size to be big, moving distance to be long. From the above, there is a possibility that we can simultaneously analysis of growth delay and body movement.

\subsection{Analysis of body movement}

Figure 7 shows analysis result by new method and previous method. According to the result by new method, amount of relative body movement was 2 pixels. In previous result, body movement was about 8 pixels. New result is clearly different form previous result. We investigated following things. First, whether we could accurately find center of gravity. Second, influences of blood vessels. Third, orbits of head and center of gravity.

About detection of center of gravity, we confirmed that correctness of center of gravity we found. Fig.8 show part of analysis data. Fig.9 shows embryo's center of gravity at

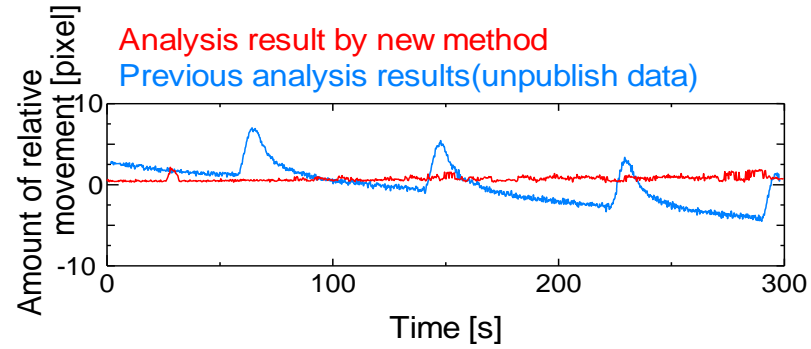

Fig.7. Comparison of analysis data

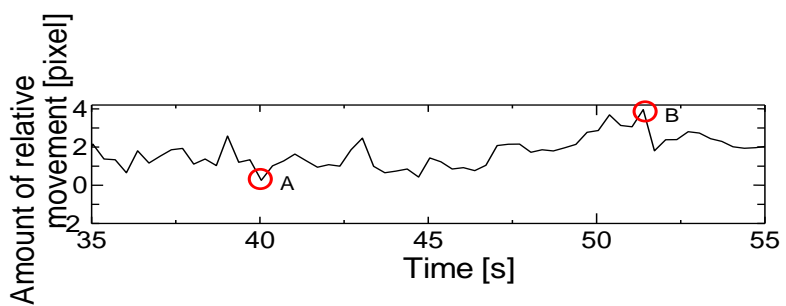

Fig.8. Part of analysis data by new method

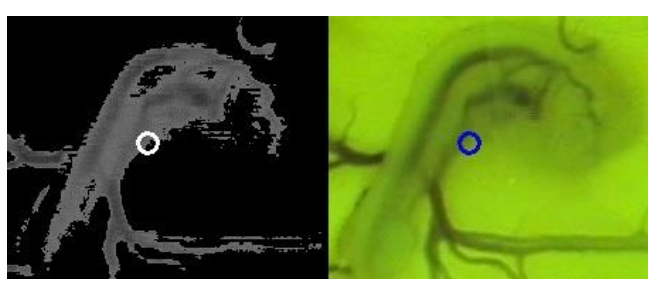

A : Coordinate of center of gravity $(357,157)$

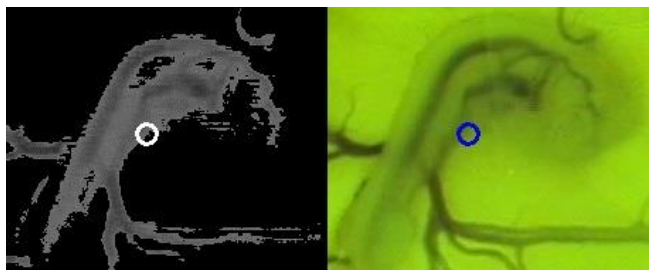

B : Coordinate of center of gravity $(360,154)$

Fig.9. Time variation of center of gravity position

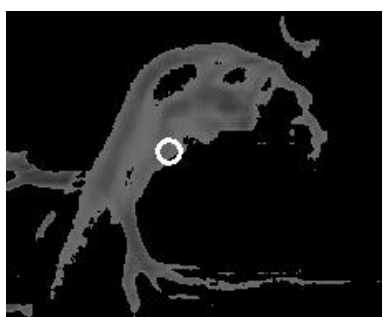

$(98,84)$

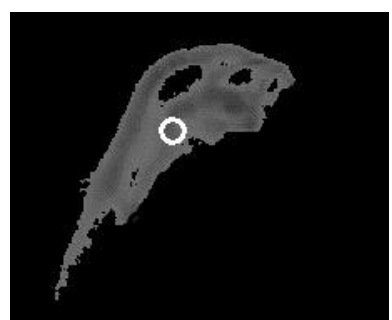

$(97,71)$
Fig.10. Effects of blood vessels on detect of center of gravity

time A and B in Fig.8. Position of embryo's center of gravity looks like it has not changed. Detected center of gravity is correctness.

Next is about effects of blood vessels. When detected 


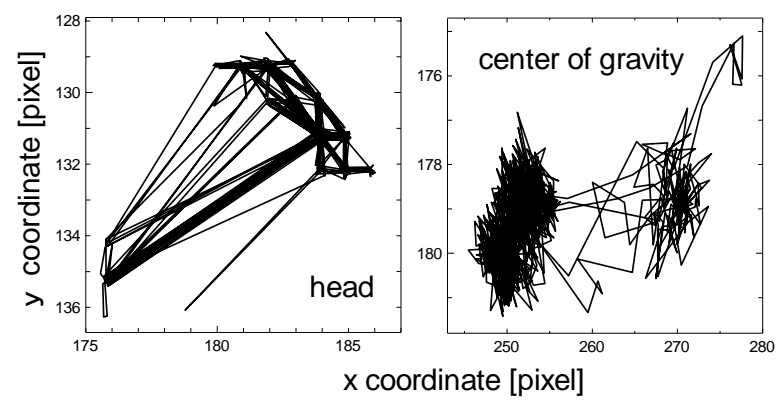

Fig.11. Head and center of gravity locus

of contour, blood vessels were detected at a same time as chick embryo. We deleted manually blood vessels and detected center of gravity. Fig.10 shows result. Center of gravity position changed and looks like approached to center of embryo. From now on, improvements require that only embryo is detected.

About locus of head and center of gravity, we made a hypothesis that embryo move around center of gravity because center of gravity was detected correctness. Thus, we surveyed locus of head and center of gravity. Fig.11 show locus of head and center of gravity. Head moved periodically. On the other hand, center of gravity moved hardly. From the above, it can be said that chick embryo's body movement is motion around center of gravity. Therefore, analysis of body movement using center of gravity detection by image processing is difficult.

\section{Conclusions}

In this research, we tried growth analysis and improvement of body movement program.

\subsection{Analysis of growth}

It found out that chick embryo's growth is delayed in hypoxic environment and when body movement becomes active, also chick embryo grew up rapidly. It may be able to simultaneous analysis of growth delay and body movement.

\subsection{Analysis of body movement}

We tried analysis of body movement using center of gravity detection. Result, it found out that embryo's center of gravity moved hardly. We think that embryo move around center of gravity. From the above, analysis of body movement using center of gravity detection by image processing is difficult.

\subsection{Future Research}

The example of embryonic growth is shown in Fig.4.
However, this result was obtained from only one individual in each $\mathrm{O}_{2}$ oxygen concentration. Therefore, more experiments should be required to verify whether this tendency of embryonic growth is general or not. In addition, this measurement system has to find out embryo manually. This procedure was needed a long time. Therefore, we aim to develop the system that can automatically find out an embryo in the next step.

In the analysis of body movement, the center of gravity was not detected accurately because blood vessels of embryo were detected simultaneously when system detected the center of gravity, which means we have to extract only embryonic body by the image processing.

\section{Acknowledgment and Additional Statement}

A part of this study was supported by a Grant-in Aid for Scientific Research (K.M. No. JP16K06397) from the Japan Society for the Promotion of Science.

This research was approved as making adequate provisions for the safety and privacy of subjects by the Life Ethics Committee of National Institute of Technology, Hakodate College.

\section{References}

(1) Yoneta, H., Akiyama, R., Nakata, W., Moriya, K. and Tazawa, H. Video analysis of body movements and their relation to the heart rate fluctuations in chick hatchlings. New insights into fundamental physiology and peri-natal adaptation of domestic fowl, pp.57-68. 2007.

(2) K. Moriya, K. Nabeshima, M. Kudou and R. Akiyama. Investigations of body movements in early stages of chick embryos using image processing. Proceeding of The $4^{\text {th }}$ combined workshop on fundamental physiology and perinatal development in Poultry. p.29,2009.

(3) Kenji Moriya, Yuya Chiba and Akito Shimouchi, Body movements during the early stages of chick embryo under intermittent hypoxic environment, Proceedings of The 3rd International Conference of Global Network for Innovative Technology (IGNITE), AIP conference proceedings; no. 1865, ISBN: 978-0-7354-1545-4, 2017 